\title{
Erratum to: Understanding Brassicaceae evolution through ancestral genome reconstruction
}

Florent Murat ${ }^{1+}$, Alexandra Louis ${ }^{2,3,4 \dagger}$, Florian Maumus ${ }^{5+}$, Alex Armero ${ }^{1}$, Richard Cook ${ }^{6}$, Hadi Quesneville ${ }^{5}$ Hugues Roest Crollius $2,3,4$ and Jerome Salse ${ }^{1 *}$

After the publication of this work [1] an error was noticed in Figure 1. There was an extra blue bar added in panel $\mathrm{a}$ in the bottom row far left. The corrected Figure 1 has now been updated in the original article. The publisher apologises for this error.

\begin{abstract}
Author details
'INRA/UBP UMR 1095 GDEC 'Génétique, Diversité et Ecophysiologie des Céréales', 5 Chemin de Beaulieu, Clermont Ferrand 63100, France. ²Ecole Normale Supérieure, Institut de Biologie de l'ENS, IBENS, Paris F-75005, France. ${ }^{3}$ Inserm, U1024, Paris F-75005, France. ${ }^{4}$ CNRS, UMR 8197, Paris F-75005, France. INRA UMR 1164 URGI Route de Saint Cyr, Versailles 78026, France. ${ }^{6}$ CNRS/UPVD UMR 5096 LGDP, 58 avenue P. Alduy, Perpignan 66860, Cedex, France.
\end{abstract}

Received: 25 January 2016 Accepted: 25 January 2016 Published online: 04 April 2016

\section{Reference}

1. Murat F, Louis A, Maumus F, Armero A, Cooke R, Quesneville H, et al. Understanding Brassicaceae evolution through ancestral genome reconstruction. Genome Biol. 2015;16:262.

\footnotetext{
*Correspondence: jsalse@clermont.inra.fr

${ }^{\dagger}$ Equal contributors

'INRA/UBP UMR 1095 GDEC 'Génétique, Diversité et Ecophysiologie des Céréales', 5 Chemin de Beaulieu, Clermont Ferrand 63100, France
}

Submit your next manuscript to BioMed Central and we will help you at every step:

- We accept pre-submission inquiries

- Our selector tool helps you to find the most relevant journal

- We provide round the clock customer support

- Convenient online submission

- Thorough peer review

- Inclusion in PubMed and all major indexing services

- Maximum visibility for your research

Submit your manuscript at

www.biomedcentral.com/submit

() Biomed Central 